\title{
Electrodes for carbon nanotube devices by focused electron beam induced deposition of gold
}

\author{
T. Brintlinger and M. S. Fuhrer ${ }^{\mathrm{a})}$ \\ Department of Physics and Center for Superconductivity Research, University of Maryland, College Park, \\ Maryland 20742 \\ J. Melngailis \\ Department of Electrical and Computer Engineering and Institute for Research in Electronics and Applied \\ Physics, University of Maryland, College Park, Maryland 20742 \\ I. Utke \\ Nanomechanics and Nanopatterning Group, EMPA Materials Science and Technology, Feuerwerkerstrasse \\ 39, CH-3602 Thun, Switzerland
}

T. Bret, A. Perentes, and P. Hoffmann

Advanced Photonics Laboratory, Ecole Polytechnique Fédérale de Lausanne (EPFL), 1015 Lausanne, Switzerland

\author{
M. Abourida and P. Doppelt \\ Centre d'Etude de Chimie Métallurgique (CNRS), 15 rue Georges Urbain, 94407 Vitry sur Seine, France
}

(Received 2 June 2005; accepted 3 October 2005; published 7 December 2005)

\begin{abstract}
Individual carbon nanotubes (CNTs) often occur in randomly dispersed two-dimensional as well as three-dimensional configurations that make device fabrication difficult. Making electrical contact to such CNTs is of practical interest. To this end, we make contact to individual metallic single-walled carbon nanotubes (SWNTs) using the focused electron-beam-induced deposition (FEBID) of pure gold. The SWNTs are grown by chemical vapor deposition on a flat substrate, and the gold leads are made through FEBID using inorganic metallic precursor gas, chloro(trifluorophosphine)gold(I), or $\mathrm{AuClPF}_{3}$, in a high vacuum scanning electron microscope. The same scanning electron microscope is also used to image carbon nanotubes, allowing for simultaneous alignment. We find equivalent one-dimensional resistivities for the SWNTs of $10-15 \mathrm{k} \Omega / \mu \mathrm{m}$ for both FEBID gold leads and leads deposited using conventional electron-beam lithography (EBL) and thermal evaporation of gold, suggesting similarly low contact resistances. We use electrostatic force microscopy to verify quantitatively similar contact resistances for one nanotube sample, $10( \pm 6)$ and $54( \pm 6) \mathrm{k} \Omega$, for FEBID and EBL leads, respectively, with most voltage dropping across the long metallic SWNT. (C) 2005 American Vacuum Society. [DOI: 10.1116/1.2130355]
\end{abstract}

\section{INTRODUCTION}

Carbon nanotube $(\mathrm{CNT})$ devices $^{1-3}$ typically rely on conventional electron-beam or optical lithography for their manufacture. This requires nominally flat substrates for resist spinning, constraining many CNT devices to twodimensional (2D) templates; etchants can free the CNT-metal devices, but the initial template remains essentially $2 \mathrm{D}$. However, interesting new devices based on the mechanical or electromechanical properties of nanotubes ${ }^{4-7}$ or nanotubes as electrically active scanned-probe elements, will require new methods of making electrical and mechanical connections on three-dimensional (3D) structures. Of similar importance, exposure to air, water, and other gases, as well as solvents and resists, affects the substrate/CNT/contact interface and device performance ${ }^{8}$ making in situ, pristine contacts desirable to study inherent CNT behavior absent impurity effects. Focused ion beam (FIB) induced deposition ${ }^{9}$ has been used for in situ contacts to CNT devices, ${ }^{10}$ but FIB

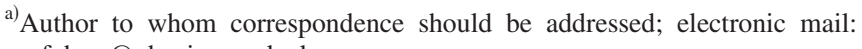
mfuhrer@physics.umd.edu damages CNTs and affects the substrate/CNT/contact interface through momentum transfer and ion implantation. ${ }^{10,11}$ Environmental electron-beam-induced deposition (EEBD) of gold-carbon composites to multi-walled nanotubes (MWNTs) has also been demonstrated in the making of in situ, 3D CNT devices with good contact resistances. ${ }^{12,13}$ However, EEBD requires high ( $>0.8$ Torr) water vapor pressure, undesirable for pristine CNT devices, and has been studied using only large-diameter MWNTs, not the more technologically interesting single-walled carbon nanotubes (SWNTs).

Here we employ focused electron-beam-induced deposition (FEBID) of gold metal lines to make contact to individual metallic single-walled carbon nanotubes SWNTs using an inorganic metallic precursor, chloro(trifluorophosphine)gold(I) or $\mathrm{AuClPF}_{3}$, in high vacuum. ${ }^{14,15}$ FEBID is a direct-writing method of making electrical contacts using a scanning electron microscope and a variety of metalcontaining precursor gases. Some current uses include in situ fabrication of magnetoelectrical devices ${ }^{16}$ and nanoscopic four-point thermal measurements. ${ }^{17}$ We find SWNT/FEBID 
gold devices have equivalent one-dimensional (1D) resistivities to SWNT devices with gold contacts deposited using conventional electron-beam lithography (EBL). Electrostatic force microscopy is used to directly measure the contact resistance between FEBID gold deposits and SWNTs, which is found to be comparable to or less than the contact resistance of electron-beam lithography (EBL) defined electrodes on the same SWNT.

\section{EXPERIMENT}

\section{A. Carbon nanotube growth}

Using a technique following Ref. 18, we first deposit carbon nanotubes using chemical vapor deposition. The starting substrate is $500 \mathrm{~nm}$ of wet-oxidized $\mathrm{SiO}_{2}$ on degenerately doped silicon where the silicon acts as a universal back gate allowing determination of metallic or semiconducting SWNTs after device manufacture. We disperse catalyst particles by dipping chips in a solution of ferric nitrate and isopropyl alcohol followed by a dip in hexane. This precipitates the ferric nitrate and leaves a random distribution of iron nitrate particles on the surface. The chips are then heated under argon to $900{ }^{\circ} \mathrm{C}$ in a tube furnace. Following Ref. 19, growth gases (ethylene/methane/hydrogen at $\sim 100 / 1300 / 2000 \mathrm{sccm}$ respectively) are then turned on for 10 min after which the gases are turned off, and the chips are allowed to cool under argon. This chemical vapor deposition growth tends to deposit long $(\sim 1-300 \mu \mathrm{m})$, randomly positioned SWNTs (diameter: $\sim 1-3 \mathrm{~nm}$ ). ${ }^{18,19}$

\section{B. Conventional lithography and deposition of gold}

Following SWNT growth, we use conventional EBL and lift-off methods to deposit chromium/gold bonding pads and leads on top of and near to SWNTs. The pads and leads are thermally evaporated from $99.99 \%$ pure gold to typical thickness of 50-70 nm after deposition of a $2-3 \mathrm{~nm}$ chromium adhesion layer. The geometry of the pads and leads together tends to incorporate 3-20 SWNTs per $1 \mathrm{~mm}$ $\times 1 \mathrm{~mm}$ area that also includes 16 different leads attached to large bonding pads. We then typically image the SWNTs using both scanning electron microscopy (SEM; Philips XL30 or Zeiss DSM982) to determine SWNT locations, and atomic force microscopy (Digital Instruments Dimension 5000 or JEOL JSPM-4210) for SWNT locations and diameters, before FEBID. Because FEBID entails simultaneous alignment with imaging and deposition happening concurrently, these steps may be either omitted or completed after SWNT device manufacture using FEBID.

\section{Focused electron beam induced deposition of gold}

We continue with the FEBID of gold leads to connect SWNTs to the EBL-defined gold leads, shown in Fig. 1. Following Ref. 14, the sample is inserted into a scanning electron microscope dedicated for FEBID (Cambridge S100). A small syringe-like reservoir of chloro(trifluorophosphine)gold(I), $\mathrm{AuClPF}_{3}$, is inserted into the SEM chamber. Deposition is done at room temperature, avoiding usage of

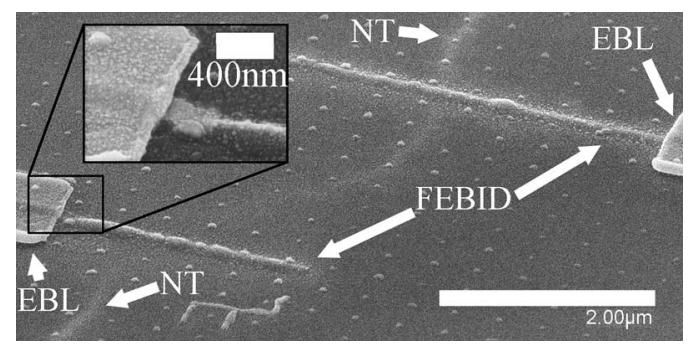

FIG. 1. Carbon nanotube with focused electron beam induced deposition (FEBID) of gold lines on $\mathrm{SiO}_{2}$. Large leads ( $1 \mu \mathrm{m}$ wide; labeled "EBL") at left and right of image were pre-deposited using conventional electronbeam lithography (EBL) and thermal evaporation. (An array of gold dots defined by EBL can also be seen; it is unrelated to this experiment.) Carbon nanotube (SWNT) runs from top to bottom of image. Focused electronbeam-induced deposition (FEBID) of $\sim 150 \mathrm{~nm}$, solid gold lines (labeled "FEBID") was done in a scanning electron microscope to connect the SWNT to the EBL leads. Inset shows region of overlap between FEBID and EBL lead.

heated stages and in situ annealing. ${ }^{17,20-22}$ The precursor flux at the syringe exit was about $1 \times 10^{17}$ molecules $/ \mathrm{cm}^{2} \mathrm{~s}$ as measured from weight loss, and the backpressure during deposition was maintained at $6 \times 10^{-6}$ mbar with a cryogenic cooling system. We then image SWNTs on the insulating surface. In Fig. 1, the labeled CNT is visible at the top, bottom, and between the FEBID electrodes. It shows varying contrast due to the tilt of the SEM and the history of the beam scan; the CNT is charging and discharging the nearby substrate which causes a local dynamic voltage contrast effect. ${ }^{23}$ After finding locations for gold deposition on top of SWNTs, a lithography system controls the electron beam during FEBID. At an accelerating voltage of $25 \mathrm{kV}$ and $100 \mathrm{pA}$ beam current, the beam diameter is about $80 \mathrm{~nm}$ at the focus. The beam is scanned at $11.6 \mathrm{~nm} / \mathrm{s}$ (corresponding to a line dose about $86 \mu \mathrm{C} / \mathrm{cm}$ or $5 \times 10^{7}$ electrons $/ \mathrm{nm}$ ) across regions containing SWNTs attached to the surface, depositing solid gold leads on top of the SWNTs by stripping off all the ligands from the chloro(trifluorophosphine)gold(I) molecule releasing pure gold. ${ }^{14,15}$ In Fig. 1, these appear as $\sim 150$-nm-wide and $\sim 20$-nm-thick lines connecting the larger EBL leads with a SWNT. The inset is a higher magnification view of the region where the FEBID gold attaches to the EBL contact. While it appears that it may be disconnected, it is still electrically conducting. Additional FEBIDdeposited gold lines were also deposited between EBL contact pads without nanotubes, in order to investigate the resistivity of the FEBID gold deposit. After FEBID, the samples are removed from the SEM for further imaging and electrical transport measurements.

\section{RESULTS AND DISCUSSION}

\section{A. Electrical resistivity of FEBID deposit}

Figure 1 also allows the resolution of individual grains of gold within the FEBID deposit, more easily seen in the inset of Fig. 1. The ability to resolve individual grains with $\sim 20-50 \mathrm{~nm}$ separation indicates lack of a carbonaceous matrix typical with organometallic precursor deposition, which 


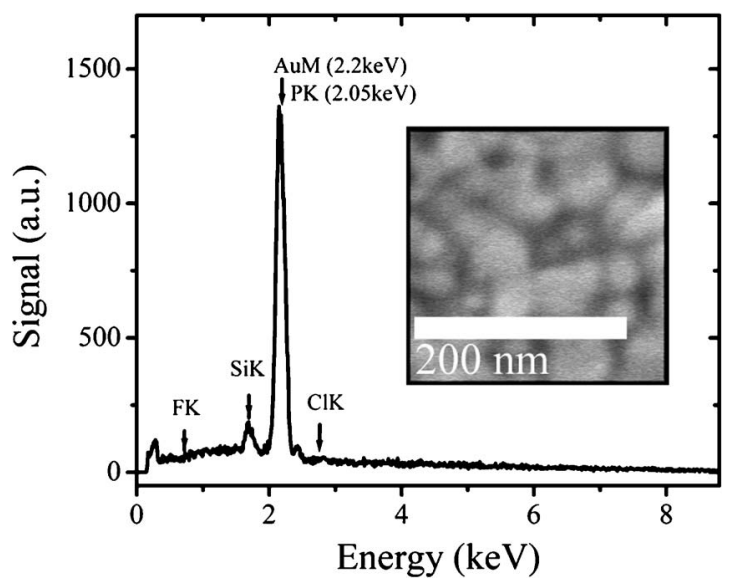

FIG. 2. Energy dispersive $\mathrm{x}$-ray analysis of focused-electron-beam-induced deposition (FEBID) of gold from chloro(trifluorophosphine)gold(I). Labeled peaks indicate the presence of gold ( $M$ line), but little to no fluorine or chlorine. The presence of phosphorous cannot be ruled out due to overlap with the $M$ line of gold. Inset is a high magnification scanning electron micrograph of individual grains of the analyzed deposition material. Such resolution $(<50 \mathrm{~nm})$ also suggests pure gold deposits.

prevents such resolution due to charging in the insulating carbon matrix. ${ }^{15}$ A separate deposition from that of Fig. 1 is featured in Fig. 2. A high-magnification image showing gold grains formed in this separate deposition is in the inset. Figure 2 also shows energy dispersive $\mathrm{x}$-ray analysis of the FEBID gold lines with strong gold $M$-line peaks at $2.20 \mathrm{keV}$ for both FEBID and EBL gold and negligible/nonexistent signatures for the nongold inorganic precursor elements, fluorine and chlorine, at 0.7 and $2.7 \mathrm{keV}$, respectively. As a phosphorus $K$ peak at $2.05 \mathrm{keV}$ would overlap the large Au band, the presence of some $\mathrm{P}$ impurities cannot be excluded, but would correspond to low concentrations $(<10 \%)$. This is further indication of solid gold FEBID leads contacting the SWNT.

Two FEBID gold lines connecting EBL pads without nanotubes were deposited at line doses of $86 \mu \mathrm{C} / \mathrm{cm}$. These lines had resistivities of $43 \mu \Omega \mathrm{cm}$ (measured by two-point contact). We note that the resistivity value obtained at low dose is about three orders of magnitude lower than in Ref. 15 due to an improved precursor supply used in the present experiments, but remains $\sim 20 \times$ larger than the resistivity of bulk or thin film gold deposited by sputtering or evaporation. This is attributed to the percolated structure of the FEBID leads, see Fig. 2.

\section{B. Nanotube two-terminal electrical transport}

After applying a bias voltage to such SWNT devices, we find one-dimensional resistivities of $\rho \approx 10-15 \mathrm{k} \Omega / \mu \mathrm{m}$ for both lithographically defined and FEBID gold leads on the same SWNT. Also, the current changes little with variations in (back) gate voltage, indicating metallic SWNTs, with no significant change in behavior for FEBID, FEBID/ lithographic, or purely lithographic leads. These values represent measurements of several contact pairs fabricated on two long carbon nanotubes. Assuming a scattering length

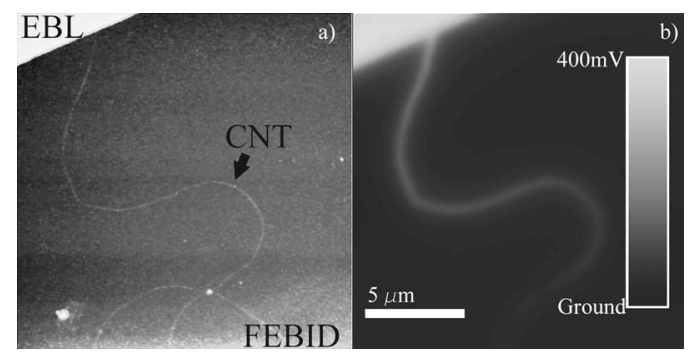

FIG. 3. Scanning probe microscopy of single-walled carbon nanotube (SWNT) device synthesized using both focused electron-beam-induced deposition (FEBID) and conventional electron-beam lithography with thermal evaporation (EBL) of chromium-gold leads. (a) Topography image showing FEBID lead on the bottom and EBL lead at top left, heights $=5$ and $20 \mathrm{~nm}$, respectively. Visible between the leads is a SWNT, height $=1.4 \mathrm{~nm}$. (b) Electrostatic potential map acquired simultaneously with (a) using electrostatic force microscopy. Brightness corresponds to potential at a given point, with a driving voltage of $400 \mathrm{mV}$ on the EBL lead.

much less than the device length and a constant intrinsic resistivity in the SWNT, this equivalence in $1 \mathrm{D}$ resistivities indicates a rough equivalence in contact resistance discussed further in Sec. III C. (The EBL lead includes a chromium adhesion layer in contact with the nanotube; however, other researchers have shown that chromium/gold contacts are similar to pure gold. ${ }^{24}$ ) We note that our $1 \mathrm{D}$ resistivities correspond to mean free paths $\ell=\left(2 G_{o} \rho\right)^{-1} \approx 0.4-0.7 \mu \mathrm{m}$, which is on order, but somewhat lower than estimates in the literature. $^{24-27}$ The lowest 3D resistivity of electron-beam deposited leads reported to date is still $5-10 \times$ that of conventional gold ${ }^{15}$ again making the equivalence of FEBID gold and EBL chromium-gold displayed here on the same SWNT worth noting.

\section{Electrostatic force microscopy}

Figure 3 displays both topography and electrostatic force microscopy images comparing FEBID contacts on a single SWNT also contacted using EBL gold leads. Figure 3(a) shows a topographical image of a SWNT, diameter $=1.4 \pm 0.5 \mathrm{~nm}$, spanning an EBL lead at top left and a FEBID lead at the bottom of the image. Figure 3(b) illustrates the simultaneously acquired electrostatic potential map using electrostatic force microscopy, a type of alternating current conducting scanning probe technique. ${ }^{26}$ Briefly, the conducting scanning cantilever responds to local changes in electrostatic potential which are being driven at the cantilever resonance by an ac bias voltage ( $400 \mathrm{mV}$ in this case). This response is detected at the photodiode of the scanning probe microscope. Drops in potential at any given point are proportional to the resistance at that point; thus, electrostatic force microscopy noninvasively determines contact resistance.

Figure 4 plots the local electrostatic potential along the length of the tube, taken from Fig. 3(b). The red triangles and black squares represent a bias being applied to either the FEBID or EBL lead, respectively. The symmetry with respect to reversal of the vertical axis in these data (as expected for an ac bias voltage) is a useful check on the linearity of the EFM potential measurement. The total 


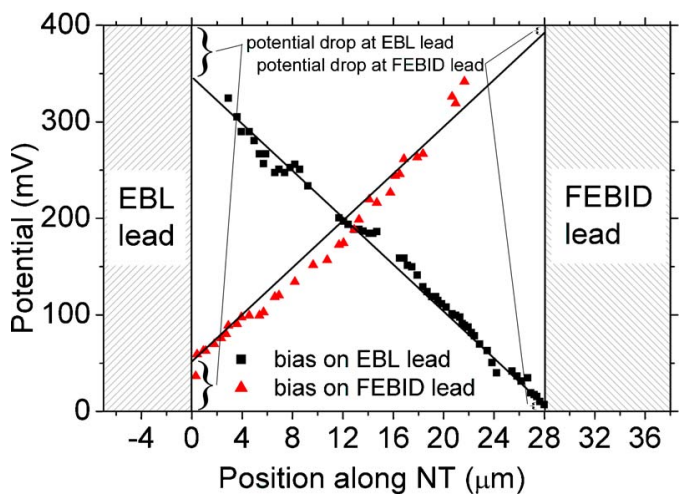

FIG. 4. Electrostatic potential along length of carbon nanotube in Fig. 3. The red triangles and black squares are for biases applied to the FEBID and EBL lead, respectively. The symmetry indicates uniform response to local potential, regardless of bias or ground lead. The drop at the leads indicates $10( \pm 6)$ and $54( \pm 6) \mathrm{k} \Omega$ contact resistances, for FEBID and EBL leads, respectively, with most voltage dropping across the long metallic SWNT, total resistance $=430 \mathrm{k} \Omega$.

resistance in this SWNT device was $430 \mathrm{k} \Omega$. The drop in potential at each of the leads quantitatively illustrates the contact resistance at that lead, $10( \pm 6)$ and $54( \pm 6) \mathrm{k} \Omega$, for FEBID and EBL leads, respectively, with most voltage dropping across the long metallic SWNT. This is further verification of the two probe measurements already mentioned, which suggest that the contact resistance between SWNTs and FEBID materials is similar to that of thermally evaporated EBL material. Of note, the contact resistance of the FEBID/SWNT junction is close to the theoretical minimum for a single SWNT contact, $1 /\left(4 G_{0}\right) \approx 3 \mathrm{k} \Omega$, where $G_{0}$ $=h / 2 e^{2}$ is the conductance quantum. Electrostatic force microscopy on the device in Fig. 1 (not shown) showed somewhat higher contact resistances $(\sim 100 \mathrm{k} \Omega)$, but again the FEBID contact resistance was less than that of EBL leads on the same SWNT.

Also of note, measurements of EEBD on MWNTs showed resistances of $9-29 \mathrm{k} \Omega$ and no correlation with length, ${ }^{13}$ indicating that the measured resistances were largely contact resistance to the (more conducting) MWNT. Such contact resistances are on the same order as in our experiments, even though the contact resistance to large-diameter MWNTs may in principle be much lower due to the larger number of $1 \mathrm{D}$ subbands available for conduction.

\section{SUMMARY}

We have shown the conductance of SWNTs contacted through FEBID gold leads to be equivalent to EBL gold leads. Although not demonstrated here, this technique allows for in situ contact to SWNTs on not only 2D substrates, but in $3 \mathrm{D}$ architectures as well. FEBID can be performed without the application of resists or solvents to the SWNT, and it does not require a separate alignment step before contact is made. While FEBID is an unlikely candidate for large-scale production of SWNT devices (due to line doses that exceed conventional EBL doses by three or more orders of magnitude), we feel its equivalence to EBL gold leads for contacting SWNTs introduces a new tool for the broad and continuing research on the physics and applications of carbon nanotube devices.

\section{ACKNOWLEDGMENTS}

This research was supported by the Laboratory for Physical Sciences and the U.S. Army Research Laboratory Power and Energy Electronics Research Program, and the Swiss National Science Foundation 200021-103333.

${ }^{1}$ P. G. Collins and P. Avouris, Sci. Am. 283, 38 (2000).

${ }^{2}$ R. Martel et al., Appl. Phys. Lett. 73, 2447 (1998).

${ }^{3}$ S. J. Tans et al., Nature (London) 393, 49 (1998).

${ }^{4}$ J. Cumings and A. Zettl, Science 289, 602 (2000).

${ }^{5}$ A. M. Fennimore et al., Nature (London) 424, 408 (2003).

${ }^{6}$ Y.-L. Li et al., Science 304, 276 (2004).

${ }^{7}$ M. Yu et al., Nanotechnology 10, 244 (1999).

${ }^{8}$ W. Kim et al., Nano Lett. 3, 193 (2003).

${ }^{9}$ J. Melngailis, J. Vac. Sci. Technol. B 5, 469 (1987).

${ }^{10}$ R. Vajtai et al., Smart Mater. Struct. 11, 691 (2002).

${ }^{11}$ P. M. Ajayan et al., Phys. Rev. Lett. 81, 1437 (1998).

${ }^{12}$ K. Mølhave et al., Nanotechnology 15, 1047 (2004).

${ }^{13}$ D. N. Madsen et al., Nano Lett. 3, 47 (2003).

${ }^{14}$ P. Hoffmann et al., Mater. Res. Soc. Symp. Proc. 624, 171 (2000).

${ }^{15}$ I. Utke et al., J. Vac. Sci. Technol. B 18, 3168 (2000).

${ }^{16} \mathrm{G}$. Boero et al., Appl. Phys. Lett. 86, 042503 (2005).

${ }^{17}$ K. Edinger et al., J. Vac. Sci. Technol. B 19, 2856 (2001).

${ }^{18}$ W. Kim et al., Nano Lett. 2, 703 (2002).

${ }^{19}$ S. Huang et al., J. Am. Chem. Soc. 125, 5636 (2003).

${ }^{20}$ A. J. DeMarco and J. Melngailis J. Vac. Sci. Technol. B 19, 2543 (2001).

${ }^{21}$ H. W. P. Koops et al., J. Vac. Sci. Technol. B 14, 4105 (1996).

${ }^{22}$ G. C. Gazzadi and S. Frabboni, J. Vac. Sci. Technol. B 23, L1 (2005).

${ }^{23}$ T. Brintlinger et al., Appl. Phys. Lett. 81, 2454 (2002).

${ }^{24}$ J.-Y. Park et al., Nano Lett. 4, 517 (2004).

${ }^{25}$ B. Gao et al., Phys. Rev. Lett. 92, 216804 (2004).

${ }^{26}$ A. Bachtold et al., Phys. Rev. Lett. 84, 6082 (2000).

${ }^{27}$ A. Javey et al., Phys. Rev. Lett. 92, 106804 (2004). 